\title{
Genetic Algorithm and Probabilistic Neural Networks for Fingerprint Identification
}

\author{
Dhia A. Alzubaydi \\ Associate Professor \\ Computer Sc. Dept. \\ AL-Mustansiriyah University
}

\author{
Thikra M. Abed \\ Researcher \\ Computer Sc. Dept. \\ AL-Mustansiriyah University
}

\begin{abstract}
Existing security methods rely on knowledge based on approaches like password or token based on approaches like access cards. Such method is not very secure, biometrics such as fingerprint, face and voice offer means of personal identification and provide increased security because they rely on who we are. In this paper, algorithm fingerprint identification is introduced. The proposed algorithm has used 196 fingerprint image back to the twenty-eight individual 140 from them has been used for training and 56 image has been used for testing .Discrete Cosine Transform has been used to extract distinctive features from fingerprint image and genetic algorithm has been used as feature selection technique .Genetic algorithm has helped to produce GA filter in order to select subset of features out of DCT. When testing the proposed system by using Probabilistic Neural Network has found the identification rate reaching to $91 \%$. This rate has emboldened on attempted using more one filter of genetic algorithm , the result that reached to $98 \%$ as identification rate with more reduction in number features.
\end{abstract}

\section{General Terms}

Pattern Recognition, Fingerprint classification and Identification.

\section{Keywords}

Fingerprint Identification, Fourier Transform, Contrast Limited Adaptive Histogram Equalization, Morphological Methods, Poincare index, Discrete Cosine Transform ,Genetic Algorithm, Probabilistic Neural Network.

\section{INTRODUCTION}

The use of fingerprints has several advantages over the other methods, and therefore is one of the most researched and mature fields of authentication. The uniqueness of fingerprints has been studied and it is well established that the probability of two fingerprints matching is vanishingly small. The probability that two fingerprints are alike is 1 in $1.9 \times 10^{15}$. Furthermore, unlike faces and voice prints, fingerprints are persistent with age and cannot be easily disguised [1].

\section{TECHNIQUES FOR ENHANCEMENT}

Image enhancement approaches fall in to two broader categories namely spatial domain methods and frequency domain methods. The approaches in the spatial domain are based on direct manipulation of pixels in an image and the approaches in the frequency domain refer to the modification of the Fourier Transform of an image [2].

- Spatial Domain Methods
The value of a pixel with coordinates $(\mathrm{x}, \mathrm{y})$ in the enhanced image $\mathrm{F}$ is the result of performing some operation on the pixels in the neighborhood of $(x, y)$ in the input image, $\mathrm{F}$. Neighborhoods can be any shape, but usually they are rectangular. Gray level transformations, Histogram processing, Enhancement using Arithmetic/Logic operations, Smoothing filters and Sharpening filters are some of the methods used in the spatial domain for image enhancement [2] .

Histogram equalization is a general process used to enhance the contrast of images by transforming its intensity values. As a secondary result, it can amplify the noise producing worse results than the original image for certain fingerprints. Therefore, instead of using the histogram equalization which affects the whole image, CLAHE (contrast limited adaptive histogram equalization) is applied to enhance the contrast of small tiles and to combine the neighboring tiles in an image by using bilinear interpolation, which eliminates the artificially induced boundaries [3] .

\section{- Frequency Domain Methods}

Image enhancement in the frequency domain is straightforward. The Fourier transform of the image to be enhanced is computed, multiply the result by a filter and take the inverse transform to produce the enhanced image [2].

\section{FINGERPRINT SEGMENTATION}

The fingerprint area contains information that may be useful in many fingerprint processing stages, while the rest of the image might contain relatively useless information and unwanted signals, such as noise. Fingerprint image segmentation is, therefore, an important fingerprint processing step because, if done correctly, it serves to separate information from noise [4].

To extract the ROI must use two-step method. The first step is block direction estimation and direction variety check while in second step used some Morphological methods.

Many algorithms have been proposed for orientation field estimation. Among these methods, the former two approaches are used popularly. The filter-bank based approaches are more resistant to noise (including smudges, breaks and creases, etc.) than the gradient-based, but they do not provide as much accuracy as the gradient-based approaches because of the limited number of the filters. Furthermore, it is computationally expensive because the comparison of all filters' output needs to be done[5].

The word morphology signifies the study of form or structure. In image processing use mathematical morphology as a means to identify and extract meaningful image descriptors based on 
properties of form or shape within the image. Key areas of application are segmentation [6].

background has determent area and discard by using following steps :

1.Those blocks without significant information on ridges and furrows are discarded based on the following formulas:

$\mathrm{E}=\left\{2 \sum \sum(\mathrm{Vx} * \mathrm{Vy})+\sum \sum\left(\mathrm{Vx}^{2}-\mathrm{Vy}^{2}:\right)\right\} / \mathrm{W} * \mathrm{~W} * \sum \sum$ $\left(\mathrm{Vx}^{2}+\mathrm{Vy}^{2}\right)$

For each block, if blocks certainty level $\mathrm{E}$ is below the threshold, then the block is regarded as a background block.

2. ROI extraction is done by two morphological operations called "OPEN" and "CLOSE". The "OPEN" operation can expand images and the "CLOSE" operation can shrink images and eliminate small cavities. The bound is the subtraction of the closed area from the opened area. Then the algorithm throws away those leftmost, rightmost, uppermost and bottommost blocks out of the bound.

\section{FEATURE EXTRACTION}

Feature extraction is a special form of dimensionality reduction. When the input data to an algorithm is too large to be processed and it is suspected to be notoriously redundant then the input data will be transformed into a reduced representation set of features (also named features vector).

The singular points can be viewed as points where the orientation field is discontinuous, which can be classified into two types: core and delta. Core point is the inner point normally in the middle of the fingerprint. Whereas delta point is normally at the lower left and right hand of the fingerprint ,Therefore the absence of delta point is possible. In close around the core point there exists rich minutiae information than others, which is necessary for the fingerprint verification/identification.

Many proposed approaches for detecting singular point are available in the literature, and most of them are working on orientation field images. The Poincare Index method (PI) is the most famous approach of singular point detection [7].

Frequency domain is a term used to describe the analysis of mathematical functions or signals with respect to frequency, rather than time. The host image is transformed to the different domain .

Fingerprint representation in DCT is a sum of sinusoids of varying magnitudes and frequencies. The DCT is used in data compression as reconstruction of original image from frequency domain is possible with few DCT coefficients [8].

\section{FEATURES SUBSET SELECTION}

The main issues in developing feature selection techniques are choosing a small feature set in order to reduce the cost and running time of a given system, as well as achieving an acceptably high recognition rate.

Various researchers have used Genetic Algorithm (GA) as an optimization tool but most of them use GA to search the optimal technique amongst the available techniques for attribute selection [9].

The GA-based method improved the results by producing a higher accuracy and reducing the number of features required for learning the classification rules, the GA-based method proved quite effective in improving the robustness of feature selection over a range of problems .

\section{PROBABILISTIC NEURAL NEWORK}

The PNN classifier was often chosen because the training of PNN is easy and instantaneous, it allows adding or removing the data to the network without need for retraining but only new sample vectors are added into existing weights when training .So it can be used in real-time [10]. Based on these facts and advantages, PNN can be viewed as a supervised neural network that is capable of using it in system classification and pattern recognition.

\section{THE PROPOSED METHOD}

This proposed method uses an automatic direct and fast procedure in both phases, feature extraction and identification. The algorithm of this method consists of the following steps:

Algorithm 1: Feature Extraction Algorithm in Proposed Method

Step 1: Input number of images $(\mathrm{N})$,for each image do the next steps.

Step 2: Preprocess the input image:

\section{1: Enhancement.}

2.2: Segmentation.

Step 3: Feature extraction:

3.1: Detection reference point.

3.2: Determine core block.

3.3: Extract features vectors of core block.

Step 4: Feature Selection.

4.1: Passing filter produced of GA on coefficients vectors.

Step 5: Save the result that represent a final features in features file.

Step 6: Repeat phase 2...5 for all training images.

Algorithm 2 : Identification Algorithm in Proposed Method

Step1:Implement feature extraction algorithm for training images.

Step2: Input test image.

Step3: Implement feature extraction algorithm for testing image.

Step4:Traning classifier using features file and test classifier using features test image.

Step5:Identification result.

\subsection{Feature Extraction Phase for the}

\section{Proposed Method}

In this phase all fingerprint image in database has passed in several steps . Input for feature extraction phase has been the image while output has been the vectors which represent features that have been extracted from the input image. Following are the more detail description of each step. 


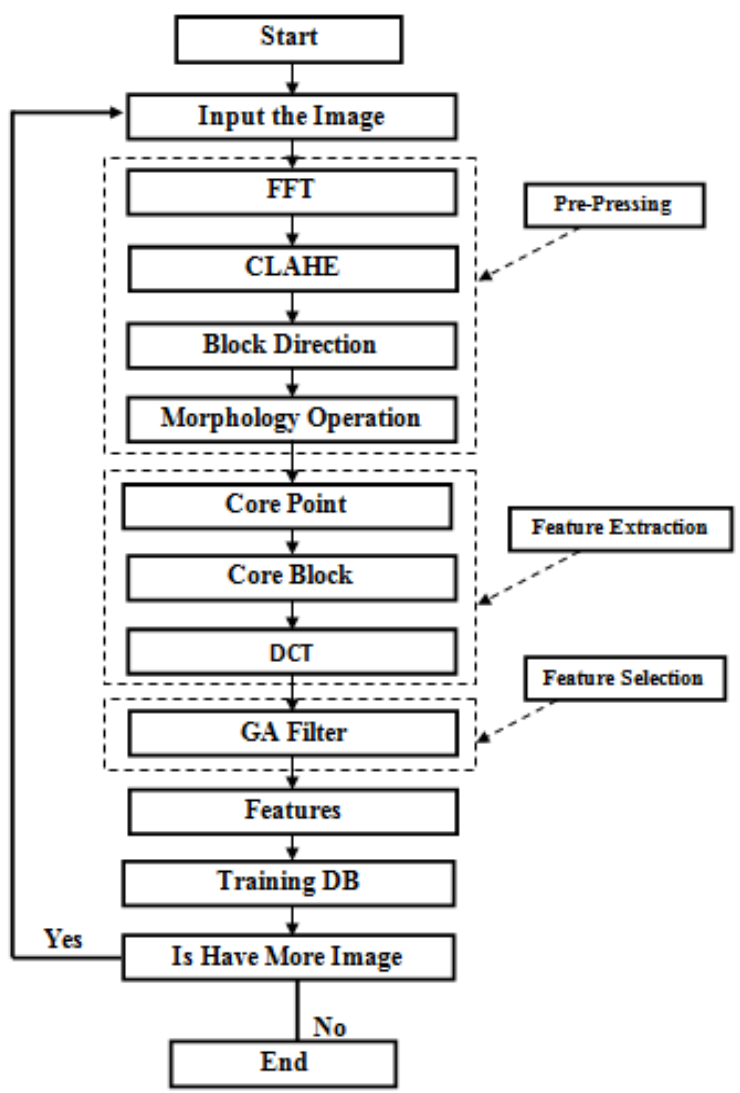

Fig 1:Block diagram of the feature extraction phase in proposed algorithm.

\subsubsection{Input the Image}

Fingerprint Images have been used acquiesced from the different types of scanners such as optical sensor, capacitive sensor or thermal sensor. The images are of poor quality ,so the enhancement step is essential.

Fingerprint images have been used as input to proposed method including seven different versions of fingerprint images per person. The Fingerprint images have used back to the twenty-eight individual, five versions of image for each person have been used as training images and the other two version of image of the same person has been used as testing images. All samples of fingerprints are of gray scale of tiff image file format.

\subsubsection{Pre-processing}

The preprocessing step is an important step for Fingerprint Identification System. This step achieves the clarity of the ridge and furrow structures in the fingerprint images. This step subdivide into enhancement and segmentation.

\subsubsection{Enhancement}

A fingerprint image may not always be in well condition due to noise that corrupts the clarity of ridges structure or basic information, which is required for fingerprint recognition. So, the enhancement step contains an enhance global and local features of fingerprints using the frequency and spatial domain technique from through :

\section{i. Ridge Enhancement}

This step has used Fast Fourier Transform (FFT) to clarify the shapes of the ridges of fingerprint images.

\section{ii. Enhance Local Contrast}

CLAHE has used to enhance the contrast of the small tiles of an image and to combine the neighboring tiles using a bilinear interpolation.

\subsubsection{Segmentation}

The goal of segmentation has extracted Region of Interest whereas the image area without effective ridges and furrows has first discarded then the bound of the remaining effective area has sketched out. ROI has determined by :

\section{i. Block Direction Estimation}

The block direction estimation operates on the gray level fingerprint image and then obtains an approximate direction for each image block. Estimation of ridge orientation values is based on gradient relationship between neighboring pixels.
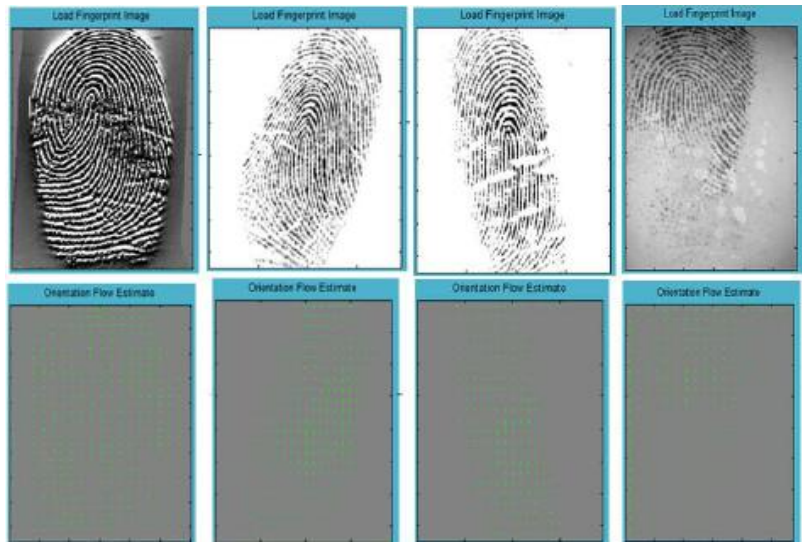

Fig $2: 1^{\text {st }}$ Row: Fingerprint images from FVC database, $2^{\text {nd }}$ Row: Image exit of orientation step.

After finishing the estimation step of each block direction , the background area has been determined and discard by using equation (1) and (close, open) operation.
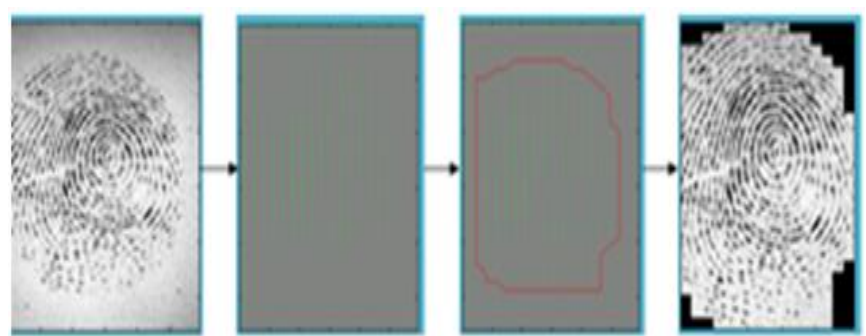

Fig 3:Show result segmentation step.

\subsubsection{Implement Feature Extraction}

Feature extraction step has focused on extracting important features of fingerprint and that require here detect core point then determine core block which has entered into DCT in order get on coefficients vectors, more details from through :

\section{i. Detection Reference Point}

After defining ROI from the previous step ,the Poincare index for each pixel of ROI has been calculated by using Poincare Index technique, determination ROI before detection 
core point has reduced probability of identifying spurious reference point.
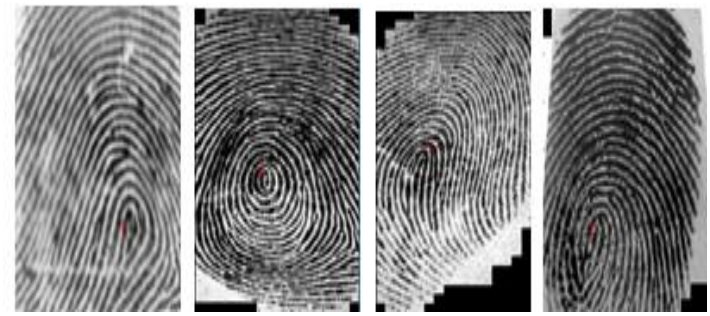

Fig4:Show result detect core point step .

\section{ii : Determine Core Block}

After a successful detect core point, the core block has been determined. The core block has extracted from area around core point and after several experimental tests have found the best size $(16 \times 16)$ and then reconstruct it as vectors in size $(1 \times 256)$. Simply ,this step has exploited an information found in the core block which surrounded core point that usually consider important information and the result has been data in the form $(1 \times 256)$ vector.
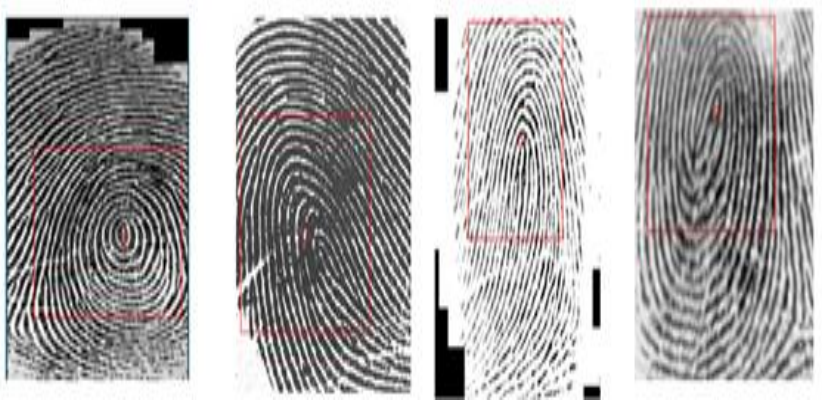

Fig 5: Result extract core block from region of interest.

\section{iii .Extract Feature Vectors}

Core block information that take from the previous step as $(1 \times 256)$ vector has entered to $1 \mathrm{DCT}$ in order extract coefficients vectors. The DCT result has got coefficients in

size $(1 \times 100)$ vector instead of each image and vectors form for each image that out of DCT as :

$\begin{array}{cccccccccc}1.1502 & -1.3348 & 0.6412 & 0.0421 & -0.3676 & 0.2775 & 0.0034 & -0.1789 & 0.1099 & 0.1156 \\ -0.2994 & 0.3119 & -0.1786 & 0.0237 & 0.0525 & -0.0441 & 0.0111 & -0.0019 & 0.0108 & -0.0048 \\ -0.0235 & 0.0412 & -0.0122 & -0.0565 & 0.1137 & -0.1129 & 0.0601 & -0.0091 & 0.0079 & -0.0490 \\ 0.0766 & -0.0453 & -0.0276 & 0.0699 & -0.0193 & -0.1152 & 0.2528 & -0.3045 & 0.2428 & -0.1186 \\ 0.0133 & 0.0265 & -0.0139 & -0.0070 & 0.0089 & 0.0002 & 0.0016 & -0.0168 & 0.0221 & 0.0063 \\ -0.0629 & 0.1128 & -0.1221 & 0.0878 & -0.0392 & 0.0095 & -0.0070 & 0.0142 & -0.0123 & 0.0030 \\ -0.0055 & 0.0324 & -0.0709 & 0.0920 & -0.0773 & 0.0374 & -0.0024 & -0.0059 & -0.0069 & 0.0172 \\ -0.0090 & -0.0101 & 0.0188 & -0.0067 & -0.0127 & 0.0156 & 0.0076 & -0.0400 & 0.0535 & -0.0356 \\ 0.0014 & 0.0207 & -0.0147 & -0.0106 & 0.0341 & -0.0450 & 0.0502 & -0.0616 & 0.0770 & -0.0778 \\ 0.0480 & 0.0055 & -0.0517 & 0.0604 & -0.0269 & -0.0230 & 0.0556 & -0.0560 & 0.0354 & -0.0140\end{array}$

Fig 6:Show core block form after passing in DCT.

\subsubsection{GENETIC ALGORITHM FILTER}

The Genetic Algorithm has been used here to generate filters. At the beginning of the Genetic Algorithm operation the choice of population size a strong interacting effect on the results. Smaller population size tends to become homogeneous more quickly. Two chromosomes have been used as population size only two filter in each generation therefore do not need to the selection techniques.
Chromosomes of a certain population size are generated, initialized with random strings, the chromosome length equal length coefficients vector $1 \times 100$.The phenotype has been real number and the genotype that using as binary number. A chromosome here is a string of bits ( 0 or 1$)$ whose size corresponds to the number of features that out from DCT. At position $i$, indicates whether the feature $i$ is selected (1) or not (0).

For better results, it is advisable to select the crossover rate quite large than the mutation rate. Crossover rate has determined equal 0 .9. The crossover method has used the one point crossover. The crossover occurs at the point of certain string length determined at random.

The mutation rate has been determined equal 0.01.The mutation operation has been applied a third order mutation, the mutation method has been used the inversion mutation.

The Genetic operations have stopped when the value fitness equal number features (number one's (1)) that specific or if the solution does not fit, the solution has stopped after 100 generation.

\section{Algorithm 3: Genetic Algorithm that used}

Step1: Determine parameter GA .

Step2: Generate randomly the first generation .

Step3: Determine stop condition .

Step4: Crossover structures .

Step5: Mutate structures .

Step6: Evaluate structures , Save filter if the result equal number of required features then break else repeat step3...6 until stop condition become true .

Filter saved in step 6 passes on coefficients vector $(1 \times 100)$ that out from DCT and has been checked if find 1 in filter has saved feature that offsets in coefficients vector but if finds 0 in filter which has not saved feature.

This method has been applied and used a different number in order to determine number one's (1) in filter which is the basis of evaluated chromosomes ; during experimentation many filters have been tested, such used 48 as number of one's (1) in filter. In addition to this, other filters have been tested with increased number one's (1) to 65,68 ,and 75 in filters that have been generated using GA and each filter has been used separately with fingerprint images.

For example :

Filter generat by GA : Filter size $(1 \times 100)$

\begin{tabular}{|l|l|l|l|l|}
\hline 1 & 0 & 0 & 1 & $\cdots \cdots$ \\
\hline
\end{tabular}

Distinctive features out of DCT :Vector size $(1 \times 100)$

\begin{tabular}{|l|l|l|l|l|}
\hline 3.3359 & 0.2739 & -0.0881 & -0.2669 & $\ldots \ldots$ \\
\hline
\end{tabular}

Features out of GAfilter step :Vector size(equal number 1 which found in GAfilter)

\begin{tabular}{|l|c|c|c|c|}
\hline $\begin{array}{l}\text { Save } \\
\text { feature }\end{array}$ & $\begin{array}{c}\text { Not save } \\
\text { feature }\end{array}$ & $\begin{array}{c}\text { Not save } \\
\text { feature }\end{array}$ & $\begin{array}{c}\text { save } \\
\text { feature }\end{array}$ & $\ldots$. \\
\hline
\end{tabular}




\subsection{IDENTIFICATION PHASE FOR THE PROPOSED METHOD}

When testing any image from 56 test image, the test image has passed in all steps mentioned in section (7.1) and after explaining the that manner gets on filter of GA and how it using, different GA filters contain different number of one's (1) have used.The number one's (1) in filters are tested $(48,65,68$ and75) training and testing images have used each filter alone in order to test the ability GA filter on improving identification rate then features out of GA filter enter to Probabilistic Neural Network

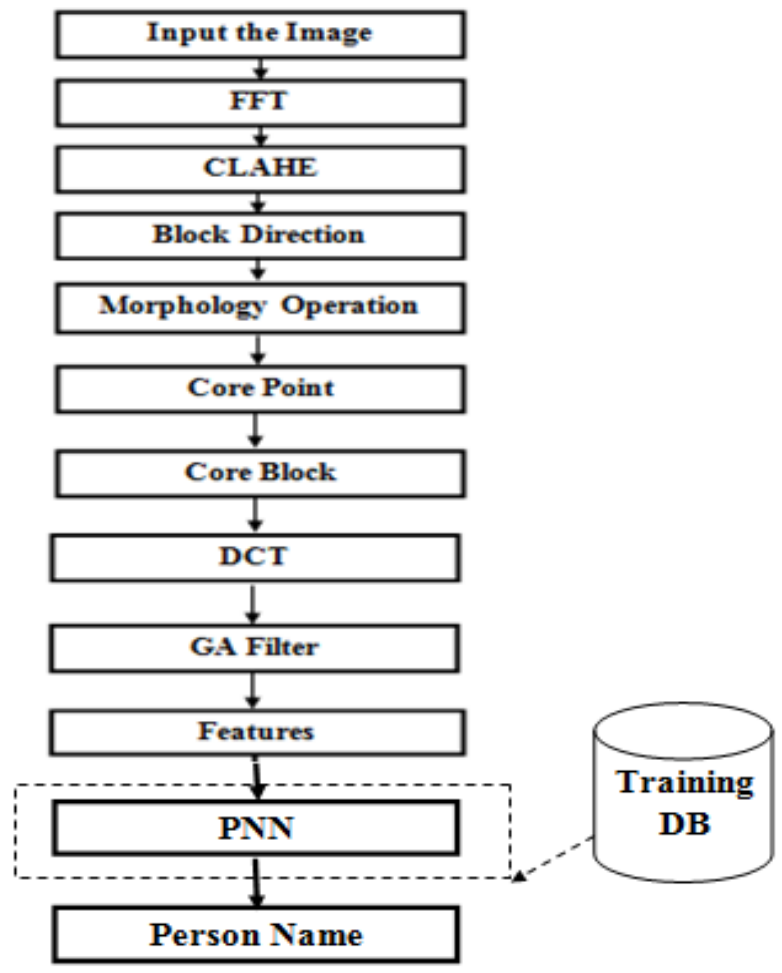

Fig 7: Block diagram of the identification phase in proposed method.

The identification results in PNN as : With 48 feature $80.35 \%$ identification rate,with 65 feature $87.50 \%$, with 68 feature $89.28 \%$ and with 75 feature $91.07 \%$ in addition all training images (140 image) correct identification.

Table 1. Summarization identification results in proposed method.

\begin{tabular}{|l|c|c|c|c|c|}
\hline $\begin{array}{c}\text { The } \\
\text { proposed } \\
\text { method }\end{array}$ & $\begin{array}{c}\text { Number } \\
\text { test } \\
\text { images }\end{array}$ & $\begin{array}{c}\text { Number } \\
\text { feautres }\end{array}$ & $\begin{array}{c}\text { Number } \\
\text { error in test } \\
\text { images }\end{array}$ & $\begin{array}{c}\text { Number } \\
\text { correct in test } \\
\text { images }\end{array}$ & $\begin{array}{c}\text { Performance } \\
\text { measure }\end{array}$ \\
\hline \multirow{2}{*}{ With PNN } & 56 & 65 & 7 & 49 & $87.50 \%$ \\
\cline { 3 - 6 } & & 68 & 6 & 50 & $89.28 \%$ \\
\cline { 3 - 6 } & & 75 & 5 & 51 & $91.07 \%$
\end{tabular}

\subsection{IMPROVEMEN ON STEP GENETIC ALGORITHM FILTER}

After explaining the proposed method and display the test results, another idea has attempted going deeply by using more one GA filter with the same number of one's (1) to get better results across improving classification. The result improvement step has been encouraging and the number of one's (1) in filters are tested (53 and 60) .In this improvement two filter are used for all images. The test results for this step disclose improving in the identification rate reach to $98 \%$, wherein use 53 feature the identification rate reach to $96.42 \%$ and when use 60 feature the identification rate reach to $98.21 \%$.

\section{CONCLUSIONS}

This proposed system has attempted the development of the fingerprint identification method depending on techniques proved efficiency such as FFT ,CLAHE , Gradient based approach , Morphology , Poincar Index, DCT, Genetic Algorithm and PNN.The test results has showed of 56 test image which back to the twenty-eight individual not found in training images,the proposed algorithm has gave identification rate reach to $91.07 \%$. Improvement step content improving for the proposed algorithm across applied two filter on fingerprint images, the test result for this step has reached to $98.21 \%$ as identification rate with use 60 feature in PNN classifier.

\section{ACKNOWLEDGMENT}

We would like to express our gratitude for our family and friends who always encouraged and supported us in all our good and bad times.

\section{REFERENCES}

[1] Pankaj, B., Kishore, B., Mohammad,N., and Mohammed, W. 2012. Fingerprint Image Enhancement And It's Feature Extraction For Recognition .INTERNATIONAL JOURNAL OF SCIENTIFIC \& TECHNOLOGY RESEARCH.

[2] T., Santhanam, and S., Radhika 2011. Application of Neural Networks for Noise and Filter Classification to enhance the Image Quality.IJCSI International Journal of Computer Science.

[3] M., Sepasian, W., Balachandran, and C., Mares 2008 .Image Enhancement for Fingerprint Minutiae-Based Algorithms Using CLAHE, Standard Deviation Analysis and Sliding Neighborhood. Proceedings of the World Congress on Engineering and Computer Science.

[4] Ishmael, S., and Mmamolatelo, E. 2011. FINGERPRINT SEGMENTATION: AN INVESTIGATION OF VARIOUS TECHNIQUES AND A PARAMETER STUDY OF A VARIANCE-BASED METHOD. International Journal of Innovative Computing, Information and Control .

[5] Jie, Z., and Jinwei,G. 2004 .A Model-Based Method for the Computation of Fingerprints' Orientation Field .IEEE TRANSACTIONS ON IMAGE PROCESSING.

[6] Chris, S.,and Toby, B. 2011 .Fundamentals of Digital Image Processing .John Wiley \& Sons, Ltd.

[7] Ali, I., and Kensuke, B. 2012. Singular Point Detection for Efficient Fingerprint Classification .International 
Journal on New Computer Architectures and Their Applications (IJNCAA), The Society of Digital Information and Wireless Communications.

[8] Lavanya, B., and K., B.Raja 2011 .Performance Evaluation of Fingerprint Identification Based on DCT and DWT using Multiple Matching Techniques . IJCSI International Journal of Computer Science.
[9] Rajdev, T., and Manu, P. 2010. Correlation-based Attribute Selection using Genetic Algorithm .International Journal of Computer Applications.

[10] Marizan, S., Adnan, H., and Zulkifilie, I. 2013.Using Probabilistic Neural Network for Classification High Impedance Faults on Power Distribution Feeders. World Applied Sciences Journal. 